University of Wollongong

Research Online

Faculty of Engineering - Papers (Archive)

Faculty of Engineering and Information

Sciences

$1-1-2012$

\title{
Continuous sorting of microparticles using dielectrophoresis
}

D F. Chen

W H. Li

University of Wollongong, weihuali@uow.edu.au

$\mathrm{H} \mathrm{Du}$

Nanyang Technological University Singapore

$\mathrm{MLi}$

University of Wollongong, ml433@uowmail.edu.au

Follow this and additional works at: https://ro.uow.edu.au/engpapers

Part of the Engineering Commons

https://ro.uow.edu.au/engpapers/4879

\section{Recommended Citation}

Chen, D F.; Li, W H.; Du, H; and Li, M: Continuous sorting of microparticles using dielectrophoresis 2012, 3035-3039.

https://ro.uow.edu.au/engpapers/4879

Research Online is the open access institutional repository for the University of Wollongong. For further information contact the UOW Library: research-pubs@uow.edu.au 


\title{
Continuous Sorting of Microparticles Using Dielectrophoresis
}

\author{
D F Chen ${ }^{1}, \mathrm{~W} \mathrm{H} \mathrm{Li}{ }^{2}$, H. Du${ }^{1}$ and $\mathrm{M} \mathrm{Li}^{2}$ \\ ${ }^{1}$ School of Mechanical and Aerospace Engineering, Nanyang Technological University, 50 Nan- \\ yang Avenue, Singapore 639798 \\ ${ }^{2}$ School of Mechanical, Materials and Mechatronic Engineering, University of Wollongong, Wol- \\ longong, NSW 2522, Australia
}

*Corresponding author email address: weihuali@uow.edu.au

\begin{abstract}
Sorting of particles such as cells is a critical process for many biomedical applications, and it is challenging to integrate it into an analytical microdevice. We report an effective and flexible dielectrophoresis (DEP)-based microfluidic device for continuous sorting of multiple particles in a microchannel. The particle sorter is composed of two components - a DEP focusing unit and a Movable DEP Trap (MDT). The trap is formed by an array of microelectrodes at the bottom of the channel and a transparent electrode plate placed at the top. The location of the trap is dependent on the configuration of voltages on the array and therefore is addressable. Flowing particles are first directed and focused into a single particle stream by the focusing unit. The streamed particles are then sorted into different fractions using the movable trap by rapidly switching the applied voltage. The performance of the sorter is demonstrated by successfully sorting microparticles in a continuous flow. The proposed DEP-based microfluidic sorter can be implemented in applications such as sample preparation and cell sorting for subsequent analytical processing, where sorting of particles is needed.
\end{abstract}

Keywords: dielectrophoresis, microfluidic sorter, particle focusing, particle sorting

\section{Introduction}

The ability to sort target biological species such as cells from the background mixture is an essential need in biomedical analysis and research. For instances, cell sorting is especially useful in immunology, where sorting is used to measure blood cell counts in patients. Cell sorting is also vital to the detection and identification of viral antigens, as well as to the development of new drug and treatment options for various diseases. The traditional cell sorter is a Fluorescence-Activated Cell Sorting (FACS) machine ${ }^{[1]}$, which is effective but expensive. In recent years, more and more biological and chemical assays are conducted in microscale devices with the rapid development of micro Total Analysis Systems ( $\mu$-TAS) or Labs-On-a-Chip (LOC) ${ }^{[2,3]}$. Microfabricated cell sorters based on miniaturized FACS approaches have been reported for sorting of cells in microfluidic channels ${ }^{[4-6]}$. These microfluidic sorters (FACS) offer certain advantages over conventional FACS such as minimal sample volumes required, inexpensive assay and less human labor involved. However, they lag significantly behind the conventional FACS in respect of separation purity and throughput. An alternative potential mechanism for microfluidics separation and sorting is the use of dielectrophoresis (DEP), a phenomenon referring to the motion of a particle in a non-uniform electric field due to the unbalanced electrostatic forces on the particle's induced dipole ${ }^{[7}$, ${ }^{8]}$. Unlike electrophoresis (EP), which affects only charged particles and relies on the charge-to-size ratio of particles, DEP affects all particles that have different dielectric properties than the medium they are suspended in. In DEP, the particle can be pushed toward/off the region of strong electric field, if the particle is more/less polarisible than the suspending medium, and are called positive DEP (pDEP) and negative DEP ( $\mathrm{nDEP}$ ), respectively. DEP has been widely used in various microfluidic devices for concentration, manipulation, separation, sorting, and transport of particles such as beads, bacteria, and cells ${ }^{[9-17]}$. Hu et al. ${ }^{[18]}$ reported a microfluidic cell sorter based on the DEP response of labeled and unlabeled cells. Kim et al. ${ }^{[5]}$ presented a multitarget dielectrophoresis activated cell sorter (MT-DACS) chip by labeling multiple cell types with unique synthetic dielectrophoretic tags that modulate the complex permittivities of the labeled cells. Due to the fact that microelectrodes are readily fabricated with conventional MEMS-based microfabrication techniques and that the generated electric field is addressable and tunable with external control, DEP represents an especially attractive and effective approach for on-chip particle manipulation. Emerging DEP-based cell sorters are more focused on the development of microdevices that are simple in design yet effective for rapid and continuous sorting of microparticles.

This paper presents a simple microfluidic device for continuous sorting of multiple particles by using movable DEP traps. Streamed particles are sorted into different fractions by the negative DEP forces from the movable trap. The particle sorter is composed of two components - a DEP focusing unit and a moving DEP trap. Injected particles in the channels are pre-focused into a streamline and then sorted by a movable, addressable DEP trap. The DEP movable trap is formed by an array of microelectrodes at 
the bottom of the channel and a transparent electrode plate placed at the top. The performance of the sorter is demonstrated by successfully sorting microparticles in a continuous flow. The sorter can also be used for sorting of multiple types $(>3)$ of particles by using a larger array. The proposed DEP-based microfluidic sorter can be implemented in applications such as sample preparation and cell sorting for subsequent analytical processing, where sorting of particles is needed.

\section{Methods and Materials}

\subsection{The Microfluidic Sorter}

The microfluidic sorter is based on the negative DEP effect for sorting of particles. Under conditions where the particles are less polarisible than the suspension medium, the particles experience negative DEP and are directed to regions of low electric field ${ }^{[7,8]}$. Fig. 1 depicts the diagrams of the microfluidic sorter. In general, the sorter is composed of an array of microelectrodes at the bottom of the channel and a conductive indium tin oxide (ITO) glass (Delta Technologies, Stillwater, MN, USA) at the top (Fig. 1b). The transparent ITO glass works as the counter electrode while it offers superior optical access. On the other hand, the use of ITO glass eliminates the need for accurate alignment encountered in making the paired electrodes ${ }^{[16]}$. The upstream focusing unit is designed to line up the particles into a single stream prior to sorting. Particles approaching the electrodes will be directed into the 'funnel' by the negative DEP (see section 'Particle Focusing'). This pre-sorting focusing step is necessary as it facilitates the subsequent sorting. Particles line up from the focusing unit and enter the sorter one by one. The sorting compartment consists of an interdigitated electrode array in the direction of the flow. By properly energizing the individual electrodes in the array and the ITO electrode, a negative DEP trap is generated over the electrode. The location of the trap is addressable by switching the voltage to the electrodes (See next section). Particles are dynamically sorted into different subpopulations by the moving trap.

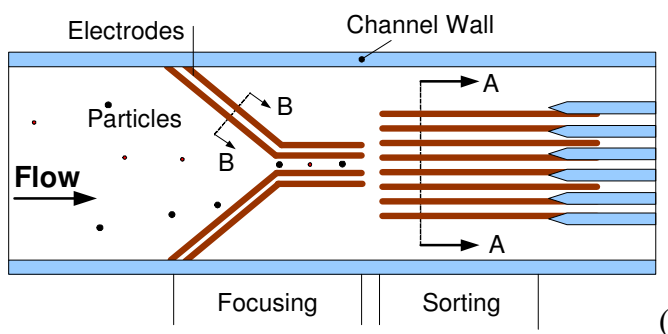

(a)

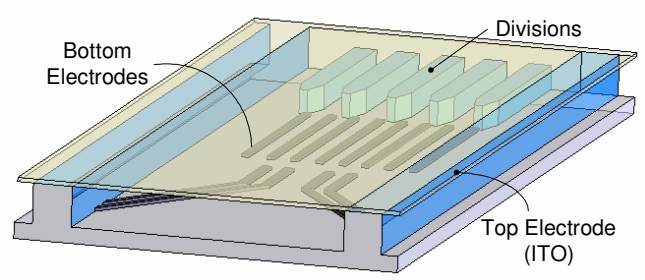

Fig. 1 Diagrams of the DEP particle sorter showing a focusing unit and a sorting unit. (a) Top view. (b) Bird's eye view. The sorter is composed of an array of electrodes at the bottom of the channel and a transparent counter electrode at the top which is formed by a piece of Indium Tin Oxide (ITO) coated glass. Wiring connections to external power supply are not shown.

\subsection{Movable DEP Trap (MDT)}

Under negative DEP, particles are trapped in regions of low electric field. The movable DEP trap (MDT) is generated by addressing the presence of low electric field in the channel. Fig. 2 shows a schematic diagram of the MDT formed over the interdigitated array. '+' and '-' denote positive and negative voltages on the electrode, respectively. In the configuration of Fig. 2a, a DEP trap is generated over the Electrode 3 where the minimum electric field is present. Then the applied voltage to each electrode switches as shown in Fig. 2b, i.e. the voltage on Electrode 3 is switched from '-' to ' + ' and the voltage on Electrode 4 is switched from ' + ' to '-'. The switch of voltage is accomplished with a control system running by LabView 8.0 (National Instruments, Austin, TX). The trap then travels to the area over Electrode 4. The movable trap enables accurate control of the particles movements. Particles can move between different trapping zones because of the movement of the trap. The trap zone extends in the direction of the flow ( $\mathrm{Z}$ direction), and the flow in turn brings the particles into the different subpopulations. To show the distribution of the electric field and the trap, simulated fields are discussed in more details in the Section 'Results and discussion'.
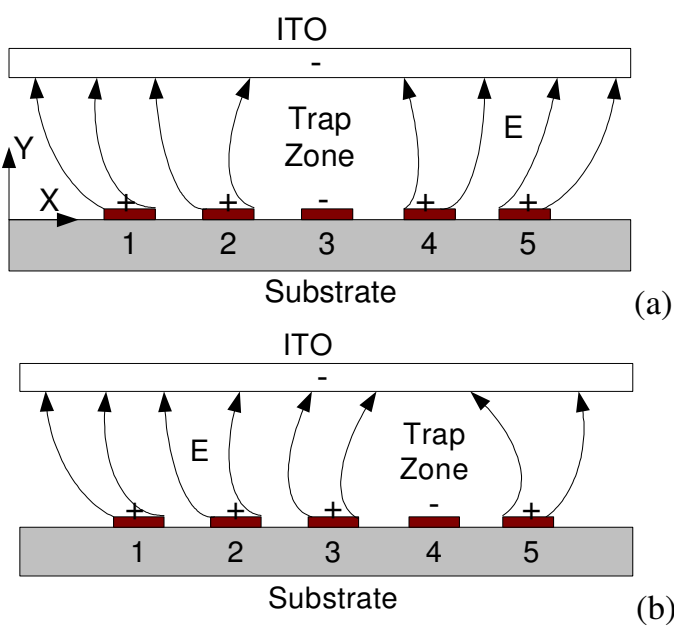

Fig.2 Principle of the DEP movable trap. Shown are the sectioned view taken from 'A-A' indicated in Fig. 1a. (a) A negative DEP trap is generated over Electrode 3 (so-called zone 3); (b) the trap is 'moved' to zone 4. '+' and '-' denote positive and negative voltages, respectively.

\subsection{Device Fabrication and Experimental Setup}

The microfluidic device containing the microelectrodes (Fig. 1b) was fabricated using conventional microfabrication techniques. The fabrication process was similar to that described by Chen et al. ${ }^{[16]}$. The electrode layer of $25 \mathrm{~nm}$ $\mathrm{Cr} / 100 \mathrm{~nm} \mathrm{Au}$ was deposited on the substrate using a magnetron sputtering process, and patterned with the lift-off technique. The substrate was a 4-inch silicon wafer with a $1 \mu \mathrm{m} \mathrm{SiO} 2$ insulation layer thermally grown on the surface. To fabricate the $600 \mu \mathrm{m}$ diameter fluidic inlet and outlet, the silicon wafer comprising the microelectrodes was 
etched through using ICP (inductively coupled plasma) deep reactive ion etching. A microchannel with $40 \mu \mathrm{m}$ in height and $800 \mu \mathrm{m}$ in width was then formed using the epoxy-based photosensitive resist SU-8 (MicroChem Corp., Newton, MA). The ITO glass was then bonded to enclose the channel using an epoxy-based adhesive. The adhesive bonding was observed to provide a bond with good coverage, high bonding strength and negligible adhesive thickness. Fig. 3a shows a photograph of the microfabricated electrodes in the channel fabricated using the above process.
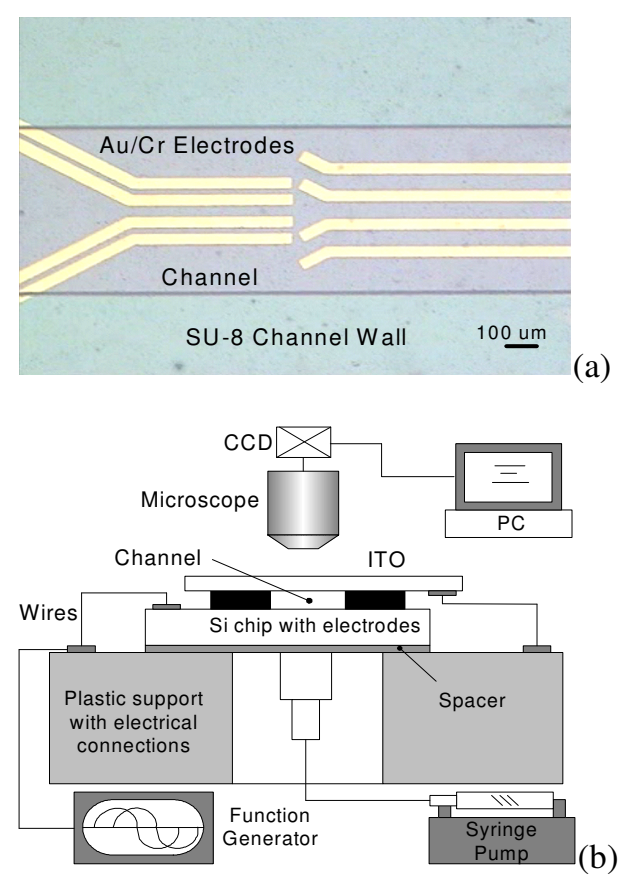

Fig. 3 (a) A graph of the microfabricated $\mathrm{Au} / \mathrm{Cr}$ electrodes at the bottom of the channel. (b) Diagram showing the microfabricated chip mounted on a support and the experimental setup composed of a microscope equipped with a CCD camera, a high frequency function generator, and a syringe pump.

The experimental setup is schematically shown in Fig. 3b. The DEP chip was mounted on a homemade plastic support on which the electrical contact pads were wired to the connection to a signal generator. Polypropylene fittings attached to the holes on the bottom slide serve as fluidic ports. One advantage of this setup is the low profile, in that there are no protrusions above the plane of the top slide, which makes it easy to mount the package under a microscope for observation. Sine wave excitation up to $20 \mathrm{MHz}$ and $15 \mathrm{Vp}-\mathrm{p}$ (into $50 \Omega$ ) was generated by a Wavetek 90 Function Generator (Wavetek Wandel and Goltermann, Inc., NC, USA). The electrical output was connected directly to the contact pads at the edge of the substrate. Fluidic connection to the chip was made via fitting and silicone tubing (Cole-Parmer, Inc. Co., IL). A syringe pump (Model NE-1000, New Era Pump System Inc., NY, USA) was used for precise control of the buffer and particle stream at a flow rate of $10-500 \mu \mathrm{l} / \mathrm{h}$. A high-resolution inspection Olympus MX40 microscope system (Olympus Corp, Tokyo, Japan) was used for particle observation. Images and movies were captured and recorded using a Sony digital color video camera.

\section{Results and Discussion}

\subsection{Simulation}

To study the generation of DEP trap and its location, the electric field was simulated with a finite-element software (Femlab 3.2, COMSOL Inc., Burlington, MA, USA). In the simulation, a model was built for the setup shown in Fig. 2. Five microelectrodes were on the bottom of the channel and an ITO glass slide was placed on the top. The bottom electrode width and the spacing between the adjacent electrodes were both $40 \mu \mathrm{m}$. The height of the channel was $40 \mu \mathrm{m}$. The voltage on the top electrode was kept constant at -5 volts, while the voltage on the bottom electrodes was switched into different configurations (A, B, C, D) as listed in Table 1. For case A, a trap was formed over the electrode 3, where the minimum electric field exists. Particles are locally trapped in the area and travel along the length of Electrode 3 with the flow. When the applied voltage switched to case B, the trap was then formed over Electrode 4 . For case $\mathrm{C}$, the trap was formed over Electrode 2. The trap can be also formed over the gap between two electrodes, as shown in case D. the location change of the trap can be readily realized by switching the voltage applied to the respective electrode. The 'movement' of the trap results in the movement of the particles across the area over the electrodes. Different particles can be sorted into different subpopulations using the movable trap.

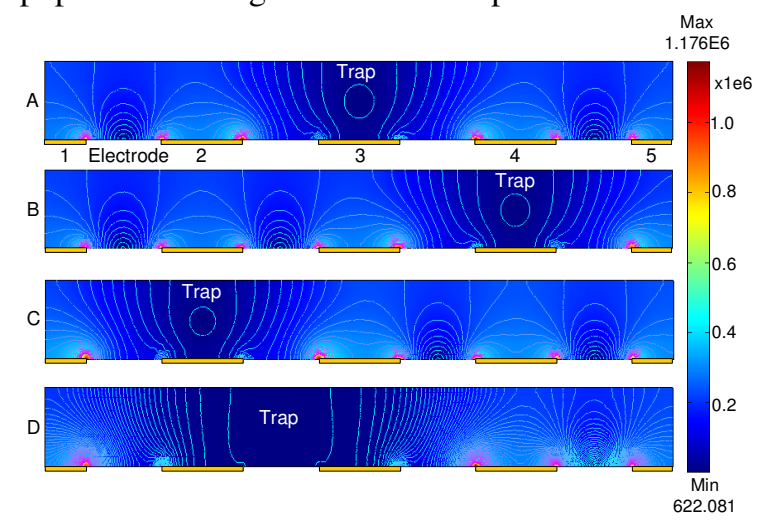

Fig. 4 Simulated electric fields for four cases (A, B, C, D) of voltages applied on each electrode as specified in Table 1.

Table 1 Voltages applied on each electrode for simulation

\begin{tabular}{ccccccc}
\multicolumn{8}{c}{$(\mathrm{V}=5$ volts $)$} \\
\hline Electrodes & 1 & 2 & 3 & 4 & 5 & ITO \\
\hline $\mathrm{A}$ & $+\mathrm{V}$ & $+\mathrm{V}$ & $-\mathrm{V}$ & $+\mathrm{V}$ & $+\mathrm{V}$ & $-\mathrm{V}$ \\
\hline $\mathrm{B}$ & $+\mathrm{V}$ & $+\mathrm{V}$ & $+\mathrm{V}$ & $-\mathrm{V}$ & $+\mathrm{V}$ & $-\mathrm{V}$ \\
\hline $\mathrm{C}$ & $+\mathrm{V}$ & $-\mathrm{V}$ & $+\mathrm{V}$ & $+\mathrm{V}$ & $+\mathrm{V}$ & $-\mathrm{V}$ \\
\hline $\mathrm{D}$ & $+\mathrm{V}$ & $-\mathrm{V}$ & $-\mathrm{V}$ & $+\mathrm{V}$ & $+\mathrm{V}$ & $-\mathrm{V}$ \\
\hline
\end{tabular}

\subsection{Particle focusing}

Particles were first focused by the funnel structure prior to entering into the sorting unit. A particle in the flow experiences both a drag force and a DEP repulsive force when it approaches the electrode, as shown in Fig. 5a. Within a proper range of flowrate, the particle will not be able to go across the electrode but move along the slanted electrode ${ }^{[16]}$. This is due to the combined effect of the two forces: DEP and hydrodynamic forces (Fig 5a). When the particle moves close to the electrode where $\mathrm{F}_{\mathrm{DEP}}=\mathrm{F}_{\mathrm{HD} 1}$, it 
moves close to the electrode where $\mathrm{F}_{\mathrm{DEP}}=\mathrm{F}_{\mathrm{HD} 1}$, it travels in the direction parallel to the length of the electrode under the effect of $\mathrm{F}_{\mathrm{HD} 2}$ (Fig. 5a). At the end, particles exit the funnel one by one to the subsequent sorting unit. Fig. $5 b$ shows the traveling of a $16-\mu \mathrm{m}$ (diameter) polystyrene particle in the focusing unit. The density, dielectric constant (permittivity), electrical conductivity and zeta potential of the polystyrene particle are $1.05 \mathrm{~g} / \mathrm{cm}^{3}, 2.4-2.7,10^{-16} \mathrm{~S} / \mathrm{m}$ and $-32 \mathrm{mV}$, respectively. In the test, the flowrate was 50 $\mu \mathrm{l} / \mathrm{h}$ from left to right. The concentration of the particles was $\sim 10^{6}$ particles/ml in DI water (PBS added) of 120 $\mu \mathrm{S} / \mathrm{cm}$. The applied voltage to counter electrodes was 15 $\mathrm{V}_{\mathrm{p}-\mathrm{p}}$ and the frequency of the voltage was $1 \mathrm{MHz}$ at which the particles experienced negative DEP. To clearly show the movement, consecutive photographs were taken and the position of the particle was incorporated into a single photo from the consecutive graphs using a software. The dotted line denotes the actual path of the particle under the experimental conditions. The particle was deflected by the slanted electrode as it came close and then moved into the 'nozzle' of the funnel. In this way, particles were focused and 'dispensed' from the nozzle to facilitate the subsequent sorting.
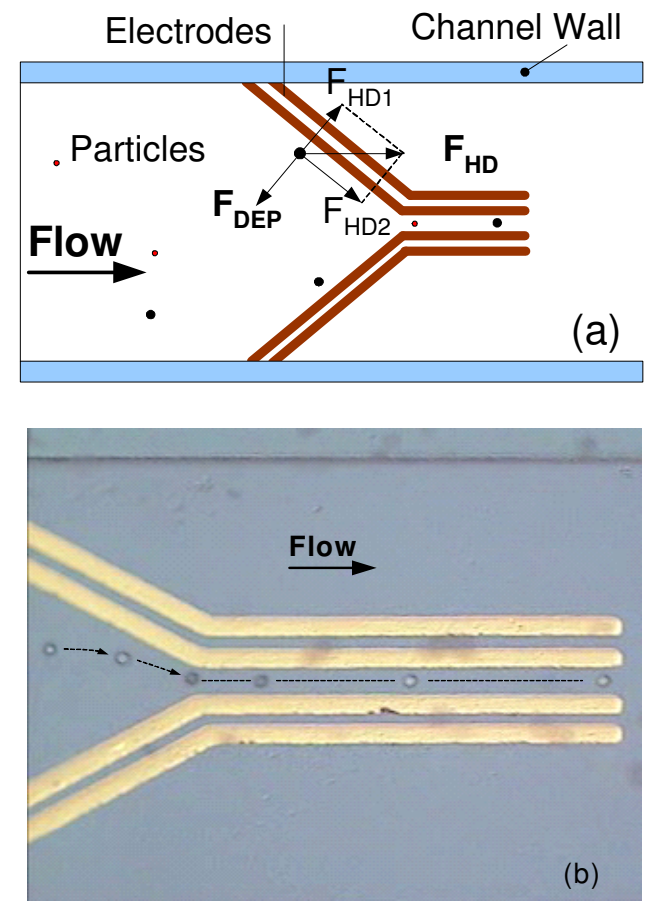

Fig. 5 (a) Particles approaching the slanted electrode experience both hydrodynamic and DEP forces. (b) A microscopic photograph showing the traveling path of a particle (16 $\mu \mathrm{m}$ diameter). The particle was deflected and moved parallel to the electrode.

\subsection{Particle sorting}

The performance of the sorter was tested in a proof-ofconcept manner by separating two types of polystyrene microspheres $(9.6 \mu \mathrm{m}$ and $16 \mu \mathrm{m})$. The concentration of the particles was $\sim 10^{6}$ particles/ml in DI water (PBS added) of $120 \mu \mathrm{S} / \mathrm{cm}$. Particles injected from the funnel were visually identified and then sorted into different branches located downstream. The sorting was performed by properly addressing the voltage on the electrodes. Fig. 6 depicts a representative result of sorting the above particles. In the ex- periment, the applied voltage to counter electrodes was 15 $\mathrm{V}_{\mathrm{p}-\mathrm{p}}$ and the frequency of the voltage was $1 \mathrm{MHz}$. The flowrate was $60 \mu \mathrm{l} / \mathrm{h}$ from left to right. The $9.6 \mu \mathrm{m}$ particles were sorted into Branch 2 using the voltage setup shown as case $\mathrm{A}$ in Table 1. Particles exited from the funnel were directed into Branch 2 by the flow (Fig. 6a). The 16$\mu \mathrm{m}$ particles were directed into Branch 3 by using the voltage setup B in Table 1. The switch between Fig. $6 a$ and $b$ was accomplished rapidly $(<1 \mathrm{~s})$ with the external control system. $100 \mu \mathrm{l}$ of sample was processed in about an hour with the current device. The device can be redesigned and used for sorting multiple ( $>3$ ) types of particles. In addition, the particles can be labeled for better selectivity during the sorting process.
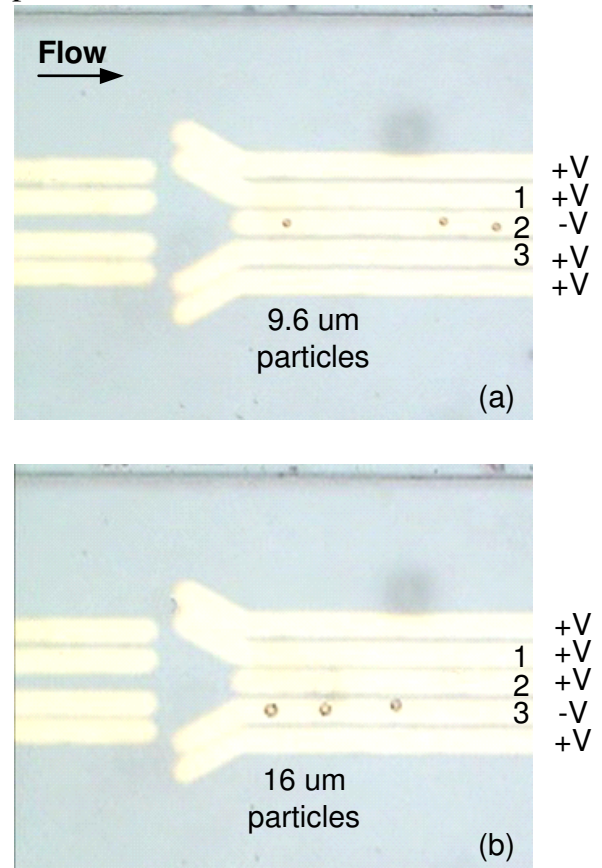

Fig. 6 A representative result of sorting $9.6 \mu \mathrm{m}$ and $16 \mu \mathrm{m}$ particles. Numbers ' 1 ' - ' 3 ' denote the branches.

\section{Conclusions}

A dielectrophoresis (DEP)-based microfluidic device for effective sorting of multiple particles in a microchannel was presented. The particle sorter was composed of a DEP focusing unit and a movable DEP trap. The MDT was formed by an array of microelectrodes at the bottom of the channel and a transparent electrode plate placed at the top. The location of the trap was addressable by switching the voltages on the electrode. The performance of the sorter was demonstrated by successfully sorting microparticles in a continuous flow. The sorter array can be re-configured for sorting more particles by increasing the number of electrodes. The proposed DEP-based microfluidic sorter can be implemented in applications such as sample preparation and cell sorting for subsequent analytical processing, where sorting of particles is needed.

\section{References}

[1] Ashcroft RG and Lopez. PA, J. Immunol. Method. 243, 13-24 (2000) 
[2] Reyes. DR, Lossifidis. D, Auroux. PA and Manz. A (2002) Anal. Chem. 74, 2623-2636.

[3] Lee. SJ and Lee SY, (2004) Applied Microbio. Biotech. 64, 289-299.

[4] Kruger. J, Singh. K, O’Neill. A, Jackson. C, Morrison. A and O'Brien. P (2002), J. Micromech. Microeng. 12, 486-494.

[5] Kim. U, Qian. J, Kenrick. SA, Daugherty. PS and Soh. HT (2008), Anal. Chem.80, 8656-8661

[6] Wolff. A, Perch-Nielsen. IR, Larsen. UD, Friis. P, Goranovic. G, Poulsen. CR, Kutter. JP and Telleman. P (2003), Lab Chip. 3, 22-27.

[7] Jones. TB (1995), Electromechanics of Particles, Cambridge, Cambridge University Press.

[8] Pohl. HA (1978), Dielectrophoresis, Cambridge, Cambridge University Press.

[9] Durr. M, Kentsch. T, Muller. Schnelle, T and Stelzle. M (2003), Eletrophoresis, 24, 722 - 731.
[10] Chen. DF, Du. H and Li. WH (2006) Sensors and Actuators A: Physical, 133, 329-334.

[11] Lapizco-Encinas. BH, Simmons. BA, Cummings. EB and Fintschenko. Y, (2004), Electrophoresis, 25, 1695704.

[12] Chen. DF and Du. H (2007), Microfluidics and Nanofluidics, 3, 603-610.

[13] Kraij. JG, Lis. MTW, Schmidt. MA and Jensen. KF (2006) Anal Chem, 78, 5019-5025.

[14] Pethig. R (1996), Crit. Rev. Biotechnol.16, 331-48

[15] Li. HB and Bashir. R (2002), Sensors Actuators B, 86, 215-21.

[16] Chen. DF, Du. H and Li. WH (2006) J. Micromech. Microen., 16, 1162-1169

[17] Chen. DF and Du. H (2010) Microfluidics and Nanofluidics, 9, 281-291.

[18] Hu. X, Bessette. PH, Qian. J, Meinhart. CD, Daugherty. PS and Soh. HT (2005), PNAS, 102, 15757 15761. 